\title{
Nouvelle recherche du modèle de " mohe » en milieu interculturel. Le cas des entreprises sino- françaises installées en Chine
}

\section{Zhijie Wang}

\section{(2) OpenEdition}

\section{Journals}

Édition électronique

URL : http://journals.openedition.org/communicationorganisation/2916

DOI : 10.4000/communicationorganisation.2916

ISSN : 1775-3546

Éditeur

Presses universitaires de Bordeaux

Édition imprimée

Date de publication : 1 mai 2004

ISSN : 1168-5549

Référence électronique

Zhijie Wang, « Nouvelle recherche du modèle de «mohe » en milieu interculturel. Le cas des

entreprises sino-françaises installées en Chine », Communication et organisation [En ligne], 24 | 2004

mis en ligne le 27 mars 2012, consulté le 19 avril 2019. URL : http://journals.openedition.org/ communicationorganisation/2916; DOI : 10.4000/communicationorganisation.2916

Ce document a été généré automatiquement le 19 avril 2019

(c) Presses universitaires de Bordeaux 


\title{
Nouvelle recherche du modèle de "mohe " en milieu interculturel. Le cas des entreprises sino-françaises installées en Chine
}

\author{
Zhijie Wang
}

1 « Mohe ${ }^{1}$ » est un ternie courant en Chine. Quand on parle d'un nouveau mariage, ce terme est souvent évoqué : les nouveaux mariés passeront sûrement une étape, courte ou longue, réussie ou ratée, de «mohe ». En chinois, mo. a le sens de moudre, de limer, de Trotter, tandis que lie évoque l'adaptation, l'harmonisation, l'union. Le mot "mohe » combine ces deux sens, exprimant ainsi tout le processus de véritable union d'un ménage.

Dans la coexistence interculturelle, il se vit aussi une ou des périodes de mohe tout comme pour un ménage. Le mohe n'y est pas un mirage à condition de faire des efforts. Le processus de mohe pourrait être une trilogie recyclable: « he " - " mo " - "lie ». Le premier «lie» est plutôt formel. "he » de capitaux, d'organisations, de personnel... L'étape de "mo » est plutôt celle de conflits, de confrontations et de conciliations. Le « he " revient après une période de « mo ", ce deuxième « he » se montre plus solide et profond sous forme de fusion.

3 La coexistence de cultures dans une entreprise n'est plus un phénomène nouveau en Chine, pays en plein développement, multipliant les joint-ventures depuis une vingtaine d'années, lin effet, avec la mondialisation, la coexistence interculturelle dans un grand nombre d'entreprises est en forte progression et les tonnes de cette coexistence évoluent au cours du temps el des expériences. Dans le cadre d'une recherche interculturelle, nous avons pu suivre l'évolution de certaines entreprises sino-françaises durant ces dix dernières années, C'est le cas, en particulier, de l'usine Dongfeng-Citröen de Wuhan. Depuis sa création, tout a changé: du niveau organisationnel aux formes de la coopération, des problèmes aux solutions, de profondeur en largeur, nécessitant de nouvelles recherches. Récemment, nous avons refait une petite enquête, dans le but de 
poursuivre son évolution, auprès des directeurs, cadres et ingénieurs français et chinois sous forme d'entretiens, de discussions libres et d'observations sur le terrain. Le présent travail vise à apporter des observations et remarques sur les évolutions rencontrées en communication interculturelle dans cette joint-venture avec vérification de l'adaptation de notre modèle " Mohe ", élaboré dans une autre recherche et rappelé très brièvement en tête de la présente contribution. Les analyses de-ces situations nous menant progressivement aux nouvelles réflexions sur la communication interculturelle plus élargie.

\section{Evolutions : Quelques faits significatifs}

En termes d'évolutions, nous ne ferons pas une énumération exhaustive des éléments interculturels, penchons-nous plutôt sur quelques faits qui nous semblent majeurs.

\section{L'organisation fonctionnelle a évolué}

Citröen-Dongleng à Wuhan est une des grandes joint-ventures sino-françaises. Economiquement, chaque côté possède $50 \%$ de capital après un stade initial de $70 \%$ pour la partie chinoise et $30 \%$ pour la partie française (dont $5 \%$ apportés par des banques françaises). De plus, l'entreprise Citröen-Dongleng s'est scindée récemment en trois entités : Dongfeng Peugeot Citroen (Fabrication) installé à Wuhan et a Xiangyang (Hubei). Dongfeng Citröen (Commercial) installé à Wuhan et Dongfeng Peugeot (Commercial) à Beijing.

6 M.Debonnet. directeur de Dongfeng Citroen à Wuhan nous a dessiné un schéma du nouvel organigramme de son entreprise :

Citroën - dongfeng commercial organisation fonctionnelle

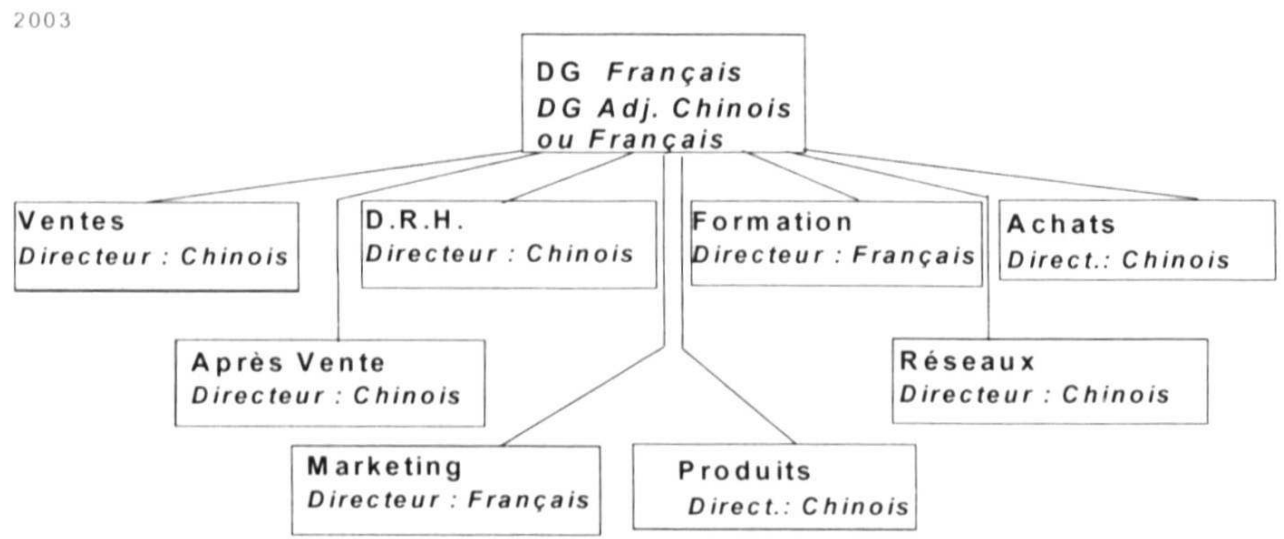

7 Cette organisation est basée non sur l'équilibre et les relations entre les deux côtés comme c'était le cas il y a dix ans, mais sur les besoins, les qualités et les compétences. On emploie les gens d'après leurs compétences et non plus d'après la nationalité ou les relations diplomatiques comme au début. 


\section{La formation préalable au départ en Chine est obligatoire}

8 Maintenant, tous ceux qui vont travailler dans des entreprises mixtes (en Chine comme en France) doivent recevoir une formation préalable durant de trois jours à trois mois : cette formation concerne généralement les présentations de contextes et les connaissances du pays intéressé, les coutumes et les rites, le savoir vivre et l'initiation à la langue. D'après les ingénieurs français, cette formation, dispensée souvent par HEC ou d'autres écoles, est essentielle. Cette initiation permet aux gens travaillant dans ces entreprises mixtes de prendre d'abord conscience de la spécificité de leur mission, d'être prêts psychologiquement à vivre les différences, et aussi d'avoir une connaissance de base sur les différences et les expériences antérieures.

\section{Les méthodes de travail sont assimilées}

9 Le costume requis, les règlements, les habitudes, les rythmes de travail el le fonctionnement de base ne posent pratiquement plus de problèmes, Tout le monde. Chinois ou français, commence le travail à 8 heures du matin. Depuis dix ans, les documents diffusés dans l'entreprise sont toujours bilingues : textes en chinois à gauche, en français à droite, bien en contraste (beaucoup d'interprètes chinois travaillent pour cela).

\section{Une normalisation des rapports est apparue}

Les directeurs et ingénieurs français et chinois, quelle que soit leur nationalité, la qualité de travail reste le seul critère pour les qualifier.

\section{Une vraie entité économique a pris naissance}

11 Dans les discussions avec les employés de cette entreprise, on ne se demande plus si c'est la partie française ou la partie chinoise qui est gagnante comme cela arrivait souvent il y a dix ans. On travaille pour «son » entreprise, Le sort de l'entreprise concerne lotis les employés, qu'ils soient français ou chinois. La naissance de celle vraie entité économique marque un pas important pour une entreprise interculturelle. Cette entité économique peut donner à l'entreprise un esprit, une matrice ou une dynamique malgré l'existence toujours évidente de deux entités culturelles.

\section{Un changement de besoins en communication}

Certains français disent : c'est presque impossible de trouver un Chinois avec qui je puisse communiquer comme avec les français, tandis qu'auparavant, on disait plutôt: il est difficile, voire impossible, de travailler avec un Chinois. Et vice versa pour les Chinois. Les points d'intérêts pour certains employés ne sont plus : comment ils (français ou Chinois) travaillent, comment ils (français ou Chinois) se comportent, mais comment ils (français ou Chinois) pensent, comment échanger des idées, C'est un défaut, mais paradoxalement on remarque là le niveau de leurs besoins en communication de plus en plus élevés : d'un niveau primaire, matériel, à un niveau spirituel ou mental. 


\section{Analyse I : De choc en conscience} étapes : connaître, comprendre, réfléchir, accepter ou non agir. La formation préalable pour les gens allant travailler à l'étranger peut les aider à passer plus rapidement les deux premières étapes, vivant plus facilement la période de «choc culturel », période inévitable d'après le modèle de Dubois ${ }^{3}$. Malgré la coexistence de dix ans pour une entreprise, l'équipe des expatriés change en général tous les quatre ans pour la partie, française, cette formation paraît donc indispensable les employés sont conscients de leur situation interculturelle: "Nous sommes prêts à vivre dans la confrontation de deux cultures, sinon, on ne serait pas venu. » disent les français. On ne trouve presque plus de choc, ou beaucoup moins, chez les français, encore moins pour les Chinois puisqu'ils connaissent déjà leurs partenaires français malgré les mutations personnelles. Les Chinois ne demandent plus à leurs collègues français quel âge ils ont ou quelle est leur situation familiale, et les français ne sont plus choqués par le chuchotement des collègues chinois pendant les réunions. Après quelques années de "Mohe », on commence à vivre dans presque une même routine : tous commencent à travailler à 8 heures du matin, les rendez-vous deviennent impératifs, on donne plus d'importance au timing le sens commercial s'ancre dans la tète du personnel... Et souvent les français avant travaillé en Chine deviennent plus souples tandis que les Chinois avant travaillé dans des entreprises interculturelles changent beaucoup leurs comportements et deviennent plus stricts et formels, par exemple.

De là, nous constatons que cette entreprise passe de la période de choc à la période de conscience. Ce petit groupe social a transité de "son royaume d'obligation", inconscient, vers «le royaume de liberté " conscient. Tandis que le modèle de "mohe» a passé plusieurs cycles évolutifs et atteint un autre niveau, plus élevé et plus profond. Les angles de chaque côté se sont " arrondis » : ce n'est plus la période des différences superficielles, premières couches de l'oignon ${ }^{4}$, mais celle où l'on va de plus en plus vers le cœur (hérosrites-valeurs). Périodes plus profondes, mais plus difficiles et plus longues. Pendant cette période, on est « sous un même toit et avec de mêmes bols. ». Cependant, cela ne veut pas dire que « l'on déguste de mêmes aliments, avec les mêmes goûts. »...

En effet les expatriés français ont après le travail, une communauté à côté avec leur école, chacun sa propre voiture, eux ou leurs femmes peuvent faire des courses chez Carrefour pas loin de chez eux (il y a actuellement beaucoup de Carrefours en Chine, trois à Wuhan ${ }^{5}$ ). Certains français (Chinois aussi) expriment les difficultés d'une communication plus profonde avec leurs partenaires : difficile d'échanger des idées. Cela parait tout à l'ait naturel puisqu'ils n'ont pas la même culture d'origine, ni la même formation, ni le même passé... en un mot, pas un même système de codage et décodage. Dans ce cas-là, la communication ne peut être que formelle et souvent superficielle. De ce point de vue, le choc culturel persiste mais avec une prise de conscience croissante vis-à-vis de celle relation interculturelle, conscience bien entendu relative. Une fusion plus profonde et générale demandant plus d'efforts et notamment de beaucoup de temps. 


\section{Analyse II : Troisième culture} un autre nom «dynamique interculturelle $»^{8}$. D'après lui : cette dynamique promeut la synergie de toutes les différences - dépasser la peur et entrer en relation avec l'autre, se transformer dans la relation sans perdre son identité, gérer malentendus et conflits en prenant en compte divers points de vue et créer des conditions nouvelles de coopération. L'objectif de cette démarche consiste à permettre la reconnaissance mutuelle, le dialogue et la rencontre, accompagner les transformations des personnes et des groupes en situation d'acculturation, et créer les conditions d'un agir commun.

Cette troisième culture ou cette dynamique, c'est la conciliation, le mélange et la fusion des deux cultures dans un contexte défini, un organisme, une entreprise en est l'exemple. De ce point de vue, nous trouvons que l'esprit mentionné plus haut, c'est précisément cette troisième culture proposée par Dodd, puisqu'il s'agit d'une culture nouvelle d'entreprise interculturelle. Ou au sens plus large: il s'agit là d'une dynamique interculturelle. Notre problématique est: sur quels principes sont fondés cette conciliation et ce mélange, ainsi que la naissance de cette troisième culture ? Pourquoi les Chinois acceptent-ils l'habitude de rendez-vous des français? Pourquoi les français commencent-ils leur travail aussi tôt (à 8 heures au lieu de 9 heures comme d'habitude) ?

Communication et organisation, 24 | 2012 
Le choc de deux cultures dans l'évolution de l'humanité a passé trois périodes, ou revêt trois formes différentes: le premier cas, c'est une culture qui remplace une autre, le deuxième, c'est une culture qui domine une autre, le troisième, c'est une coexistence pacifique et amicale des deux cultures. Au XXI siècle, le troisième choix semble s'imposer naturellement bien que l'hégémonisme culturel trouble toujours les esprits, en particulier dans le cadre actuel de la mondialisation («La communication monde» de Mattelart a bien traité ces problèmes tout en critiquant l'hégémonisme culturel. $\left.{ }^{9}\right)$. Notre postulat de base reposant sur l'égalité des cultures les unes par rapport aux autres nous porte évidemment sur ce choix.

Il est évident que dans cette coexistence interculturelle pacifique et amicale, il ne manque pas de "mohe », donc de confrontations, de compromis et de conciliations. La base de ce «mohe » doit se fonder non sur les forces, ni sur la volonté de certains, mais sur d'autres principes loyaux et raisonnables. En évoquant l'histoire de la naissance des cultures, nous remarquons que "qualité » et " contexte » paraissent importants comme principes dans la confrontation des cultures: qualité - les bonnes qualités pourraient toujours être appréciées et remplacer les défauts, contexte - l'environnement conditionne beaucoup le fonctionnement.

Le principe de «qualité » parait primordial. L'histoire de cultures est remplie de fusions, de mélanges et d'emprunts, chaque culture intègre beaucoup d'éléments et facteurs "étrangers ", mais on garde et inspire toujours de bons usages et qualités d'une culture tout en négligeant le reste.

Les Chinois ne gardent plus leurs anciennes robes longues traditionnelles parce qu'elles ne sont pas pratiques;

Les Européens prennent plus de thé et de soja parce que ces aliments sont utiles à la santé

Les cultures évoluent par la complémentarité, la sélection et l'absorption des qualités des autres :

La culture française ne manque pas de traces des Gaulois, des Celtes et des Romains : la chinoise de Hans, de Mongoles, de Mandchous.

La culture japonaise comprend beaucoup de traces chinoises;

La culture arabe s'imprègne de beaucoup d'autres, asiatiques et européennes :

La Grèce s 'est inspirée de l'Égypte et Rome a tiré profit de la Grèce...

Gérard Leclerc a fait un bilan de cette histoire mondiale d'emprunts et de mélanges dite premièrement «La mondialisation culturelle $»^{10}$. À l'heure actuelle, cette "mondialisation culturelle » est beaucoup plus marquée par «les flux de marchandises - le commerce mondial, les flux de capitaux - la globalisation financière, les flux de personnes - les immigrations internationales et les flux d'informations - la mondialisation culturelle $»^{11}$. Ces flux enrichissent naturellement chaque culture, et créent d'autre part de plus en plus de points communs entre cultures.

41 Pour une entreprise, une bonne habitude de travail doit et peut toujours être conservée quelle que soit son origine de quel pays ou de quelle culture, par exemple, bien à l'heure, bien précis, efficace, travailleur...

Quand il s'agit d'une entreprise interculturelle installée dans un des deux pays, le principe de « contexte » reste aussi important malgré deux cultures juxtaposées, puisque l'environnement du pays où s'installe l'entreprise conditionne grandement sa réussite. 
Pour un meilleur fonctionnement, on doit observer les lois et les circonstances relatives au pays.

Ces deux principes semblent assez bien s'appliquer chez Dongfeng-Citröen. Le " mohe » bien réussi dans la coexistence interculturelle a confirmé ces deux principes. La « troisième culture » se créée aussi à partir de ces deux principes.

Mais en ce qui concerne cette troisième culture, il ne s'agit pas là de l'établissement d'une culture générale ou universelle. La troisième culture n'efface pas l'existence des deux cultures d'origine, au moins pour l'instant. La troisième culture ne signifie pas la perte d'identité de chaque culture, mais un bon débouché pour la confrontation des deux. En réalité, cette notion de «troisième culture » est historique et relative par rapport aux deux cultures intéressées, pour une période définie et dans un contexte limité, différente de celle des deux cultures composantes. Par exemple, pour Citröen-Dongfeng, c'est plutôt une culture ni chinoise ni française, mais une culture spécifique à Citröen-Dongfeng sinofançaise.

Dans la «société » Citröen-Dongfeng, groupe d'individus interculturels, cette troisième culture reste bien limitée et bornée, mais est-ce qu'elle ne sera jamais élargie dans le futur pour devenir un champ d'expériences, de transition, voire l'ébauche d'une globalisation plus profonde et plus élargie de la culture de ce groupe social?

\section{Analyse III : « Inter » et « intra »}

La coexistence chez Citröen-Dongfeng nous découvre un autre fait: dans un premier temps de la coopération, les problèmes interculturels paraissaient plus fréquents et évidents : au fur et à mesure, les problèmes inter et intra se confondent et se croisent de plus en plus. Par exemple, au début, l'interculturel est pour certains, le seul critère d'explication à toute contrainte et à tout problème: Il ne m'a pas compris parce qu'il est Chinois... C'est difficile île communiquer avec lui car il est français...

Mais avec le temps, les remarques sont différentes, plus nuancées : les appréciations ne sont plus marquées par la nationalité, mais par la personnalité : Ce jeune est sérieux, bien différent de l'autre, je suis satisfait de son travail... J'apprécie beaucoup ce vice-directeur, on s'entend bien entre nous...

À partir de là, nous essayons une approche tournante ${ }^{12}$ en ré-envisageant ces deux domaines une fois ensemble : inter et intra.

D'après la théorie de la culture d'Hofstede ${ }^{13}$, chaque individu est porteur de différents niveaux de culture : celui de la nation, del a région, du groupe, du sexe, de la génération, de l'origine sociale ou de l'organisation. Dans la communication interculturelle, on rencontre surtout des problèmes de différences de nation, de culture, mais aussi d'autres niveaux: formation, religion, sexe, génération et métier. Chaque personne relève de groupes d'appartenances culturelles diverses qui s'entrecroisent.

Les gens de cultures différentes mais de même âge. de même sexe, ou de même métier peuvent souvent plus facilement communiquer entre eux que ceux de cultures différentes et aussi de formation, de sexe et de métier différents, lit paradoxalement, les gens d'une même nation ou culture, mais de formation de sexe, d'âge et de métier différents, posent également des problèmes dans la communication. Il est indéniable que certaines personnalités de France sont pratiquement identiques à celles de Chine, et qu'on trouve 
les défauts et les qualités identiques en Asie et en Europe. La réussite de certains expatriés français ou l'échec d'autres en est une bonne preuve : certains, d'un caractère plus tolérant, peuvent plus facilement réussir, tandis que d'autres, trop rigoureux et durs rencontrent souvent des problèmes. livres chinois (et français aussi, évidemment) sur la communication. Ces stratégies pourraient faciliter la compréhension, la communication, tout en amortissant les conflits et confrontations. Elles sont pratiquées depuis longtemps dans la communication intraculturelle mais conviennent également à la communication interculturelle. Cette dernière, étant beaucoup plus récente et jeune, a besoin de tirer des expériences et des lois de la première. De plus, entre ces deux domaines, il existe beaucoup de ressemblances : les problèmes, les arts de communiquer, le processus et même certaines théories. Mohe, ce modèle même en est déjà une preuve: un phénomène de la communication intraculturelle emprunté pour une recherche interculturelle.

\section{Distinguer les problèmes différents et les traiter avec des mesures adéquates}

Dans une entreprise interculturelle, les facteurs inter et intra se croisent souvent. Il est important de les distinguer lorsqu'on traite des problèmes afin de prendre des mesures adéquates au lieu de mélanger tout. Quelquefois, la position apathique (alternative) s'avère nécessaire. Il vaut mieux éviter d'aligner tous les problèmes et tous les employés de l'entreprise dans le casier « interculturel » : tel problème, tel remède. 


\section{Prendre en compte les facteurs de tout niveau inter et intra}

61 Normalement, au début de la coopération ou de la coexistence, les problèmes interculturels se montrent plus fréquents et évidents : au fur et à mesure de «Mohe», les problèmes inter et intra apparaissent croisés et mélangés. Dans ce cas-là, la manière de considérer les choses doit aussi changer et se compléter.

\section{Conclusion}

Depuis ces dix ans, nous avons vu chez Dongfeng-Citröen de Wuhan de grands changements: l'organisation fonctionnelle évolue, l'ambiance change, l'imprégnation réciproque devient plus profonde, la fusion plus solide, les rôles des deux parties redistribués et les problèmes bien différents de ceux du départ... Pourtant les principes et les enjeux restent, les deux entités culturelles existent toujours. Les cycles du modèle de "Mohe» se sont déroulés. Ce n'est pas un modèle figé, mais plutôt un modèle toujours en recherche, à compléter et à enrichir. Après une ou des périodes de "Mohe» naît un esprit d'entreprise dit « la troisième culture », culture spécifique au milieu interculturel. Dans la coexistence interculturelle, les facteurs interculturels et intraculturels se confondent et se croisent avec son développement. L'important est de bien les distinguer et de les traiter différemment. Pour une meilleure réussite dans la coexistence interculturelle des entreprises sino-françaises, il serait utile d'emprunter les expériences el les lois de la communication intraculturelle.

Nous avons connu de grands progrès dans les entreprises interculturelles malgré les vicissitudes, et nous y attendons de meilleures perspectives en termes de coexistence. Le monde industriel et commercial sert toujours d'avant-garde dans l'évolution de l'humanité. Il représente les tendances et l'orientation du développement de la société. Il en est de même pour le domaine de la communication interculturelle. Dans la recherche du modèle de Mohe, nous remarquons également l'importance du renouvellement de notre épistémologie de la communication interculturelle : essayer de voir les choses d'une manière multidimentionnelle progressive et conjecturale. Le monde est infini, mais notre vision est restreinte. Ce que nous pouvons faire, c'est élargir nos champs de vision et connaître un peu mieux ce monde : connaître l'arbre et son environnement forestier au lieu d'agir au contraire tout comme le critique le proverbe chinois : on ne peut pas « voir l'arbre sans connaître la forêt » très proche du proverbe français: "L'arbre ne doit pas cacher la forêt ». Donc il serait intéressant que nos recherches sur la communication interculturelle se localisent non seulement sur les éléments de ce domaine, mais aussi sur ceux de la communication intraculturelle.

\section{BIBLIOGRAPHIE}

BAUDRAND. V., Les éléments clés de la mondialisation. Levallois-Perret : Studyrama., 2002. 
GREC/O, La communication du risque. Communication et organisation, 2001.

HOFSTEDE. G., Vivre dans un monde interculturel. Paris : Les Editions d'Organisation. 1994.

LARRY. A., SAMOUVAR et allii. Understanding intercultural communication. Wadsworth Publising Co. 1981.

LECLERC. G., La mondialisation culturelle. Paris : PUF. 2000.

LING. D., Etudes sur la communication interculturelle. Fujian: Editions Xiamen. 1994.

LIU S., YU. W., La communication interculturelle. Heilongjiang: Editions Heilongjiang. 2000.

MATTELART. A., La communication-monde.. Paris : La découverte. 1992 ROGERS. E.M.. STENTFATT. Intercultural communication. Waveland: Prospect Heighls.IL. 1999.

SUNZI. L'Art de la guerre de Sunzi et 36 stratégies. Laifu: Editions Huanghe. 2001.

WANG. Y., Il faut renouveler la pensée.. Beijing. Editions de Jiéfangjun. 1996.

WU. Y., La vision multidimensionnelle, Beijing : Editions de l'Université de Beijing. 2001

ZHENG. Lihua, Chine et Mondialisation. Guangzhou. 2002

\section{NOTES}

1. .Voir La communication du risque. Communication \& Organisation. GREC/O. 2001.

2. .cf. Intercultural communication. Rogers E.M et Steinfalt. Prospect Heights. IL Waveland, 1999

3. Le modèle de Dubois résume les 5 périodes suivantes «lune de miel». choc de culture première adaptation. solitude psychologique et acceptation et intégration

4. cf Vivre dans un monde interculturel d'Hofstedt. Il divise l'oignon en quatre couches symboles héros, rituels et valeurs

5. La ville de Wuhan est divisée en trois parties par les fleuves Yangsté et Hanjiang Ces trois Carrefour s'installent chacun dans une partie chronologiquement. Hanyang. Hankou et Wuchang

6. .cf. Le second souffle de l'entreprise P Kotter. James L.. Heskett Culture et Performances

7. .cf. Dynamics of intercultural communication. Dodd. CH.. Boston. MA McGraw Hill. 1998

8. . cf Acquérir une compétence interculturelle. mémoire de DESS de psychologie. Marc THOMAS Université de Nancy. octobre 2000

9. cf La communication-monde. Armand Mattelart. La découverte Paris. 1992.

10. .cf. La mondialisation culturelle. Gérard Declere. Paris FUF. 2000

11. .cf Les éléments clés de la mondialisation. Vincant Baudrand. Levallois-Perret Studyrama. 2002

12. cf. Il faut renouveler la pensée.. Wang Yin. Editions de Jiéfangjun. Beijing. 1996

13. .cf. Vivre dam un monde interculturel d'Hofstede Chapitre : Nivaux de culture

14. Traduction mot a mot zou - s'en aller, wet comme shangee la meilleure stratégie 


\section{AUTEUR}

\section{ZHIJIE WANG}

Université de Wuhan, Chine. GREC/O. Université Bordeaux 3 